\title{
INFLUENCIA DA EVOLUÇÃO COSTEIRA HOLOCÊNICA NA OCUPAÇÃO POR GRUPOS SA., MBAQUIEROS - RESULTADOS DAS PROSPECÇÕES ARQUEOLÓGICAS NAS REGIÕES LITORÂNEAS DOS ESTADOS DE SERGIPE E DA BAHIA NO NORDESTE DO BRASIL
}

\author{
LA EVOLUCIÓN DE LA COSTA EN EL HOLOCENO Y SUS \\ INFLUENCIAS PARA LA CONSTRUCCIÓN DE LOS CONCHEROS. \\ RESULTADOS DE LA PROSPECCIÓN ARQUEOLÓGICA EN LOS \\ ESTADOS DE SERGIPE Y BAHÍA EN NORDESTE DE BRASIL
}

\author{
Suely Amâncio-Martinelli, Cristiana de Cerqueira Silva Santana**, Márcia Barbosa Guimarães ${ }^{* * *}$
}

\begin{abstract}
The greatest evidence of the pre-colonial presence that occupied the coastal region are archaeological sites called coastal sambaquis. Aiming to identify potential areas for installation of sambaqui groups on the coast of Sergipe and Bahia, it was proposed to use an unusual approach in Brazilian archeology which is to use, as a starting point, an understanding of the evolution of the coastal zone in the last 5600 cal years BP, to guide the archaeological exploration in search of shell mounds sites. The prospecting work carried out resulted in the discovery of archaeological sites of the sambaqui type on the northern coast of Bahia. This study becomes relevant when enabling increase of information concerning its physical environment and the transformations experienced by the coastal zone during the Holocene and their impact on pre-colonial occupation of the region.
\end{abstract}

Key words: shell mounds, Holocene, coastal evolution.

Las investigaciones sobre el cambio de la costa este brasileña revelan que cerca de 5.600 cal b.P., el nivel relativo del mar ha hecho que los ambientes lagunares tuvieran una gran expansión, contribuyendo a la ocupación de estos ambientes por grupos precoloniales. Con objetivo de identificar concheros en la costa del Estados de Bahia e Sergipe, hemos realizado nuestras prospecciones a través de la evolución costera en los 5.600 cal b.P. La metodología, no muy usual en los estudios arqueológicos brasileños, ha resultado en los hallazgos de yacimientos concheros en el Norte del estado de Bahía. Los concheros siguen siendo investigados en sus resultados, demostrando importantes informaciones a la ocupación precolonial de la región durante el Holoceno.

Palabras claves: concheros, Holoceno, evolución costera.

\section{Introdução}

As pesquisas mostram que a costa leste brasileira, no decorrer do Quaternário, esteve submetida a importantes flutuações do Nível Relativo do Mar (Bittencourt et al. 1979, Bittencourt et al. 1983, Dominguez 1987, Dominguez et al. 1981, Dominguez et al. 1996, Suguio et al. 1985). Ainda segundo Suguio et al. (1991) e Martin e Dominguez (1992), no Holoceno, durante a Última Transgressão, ocorrida por volta de 5.600 anos A.P., os ambientes lagunares atingiram sua expressão máxima. As transgressões subsequentes há 5.600 anos A.P., alcançaram cotas abaixo daquelas verificadas durante o máximo da Última Transgressão, e desta forma, não se formaram grandes áreas protegidas na costa. Durante as regressões, por outro lado, a maioria dos ambientes lagunares, formados durante a Última Transgressão, desapareceu transformando-se em brejos e pântanos.

Fornecendo suporte a grande maioria dos estudos arqueológicos que abordam os sambaquis, testemunhos mais diretos das populações pescadoras e coletoras, os estudos geomorfológicos, sobretudo os relacionados aos níveis relativos do mar, desempenharam/desempenham papel fundamental

* Universidade Federal de Sergipe, Núcleo de Arqueologia, Programa de Pós-graduação em Arqueologia, Bolsista do CNPq, Brasil. Correo electrónico: suelyamancio@ hotmail.com

** Universidade Estadual da Bahia, Departamento de Educação, Laboratório de Arqueologia e Paleontologia, Brasil. Correo electrónico: ccsilva@uneb.br

*** Universidade Federal de Sergipe, Núcleo de Arqueologia, Programa de Pós-graduação em Arqueologia, Brasil. Correo electrónico: marcia.segal63@gmail.com.br 
na compreensão da colonização da costa brasileira durante o Holoceno (ver Scheel-Ybert. 2009).

As modificações da fisiografia e dinâmica costeiras exerceram papel importante na forma e na dinâmica da ocupação da zona litorânea por populações pré-coloniais como relatado em Jardine \& Morrison (1976), para o litoral da Inglaterra, Dunbar et al. (1991) e Erlandson (2010), para o litoral dos Estados Unidos, Sandweiss (2008), para a Costa Pacífica da América do Sul, e Amâncio e Dominguez (1999), Barbosa-Guimarães (2012), DeBlasis et al. (2007), Silva (2000), Suguio et al. (1991) e Villagrán et al. (2011), para a costa brasileira.

A ocorrência de sítios costeiros pleistocênicos é muito rara no Brasil (Figuti 1993). No entanto, a partir do início do Holoceno os registros de sítios costeiros com utilização efetiva de seus recursos ambientais são frequentes. A maior evidência da presença de grupos que ocuparam a região costeira são os sítios denominados sambaquis, berbigueiros ou sernambis (Gaspar et al. 2008). Esta denominação é dada aos sítios que apresentam diversas dimensões e uma estratigrafia composta, em sua maioria, por conchas, sendo constituídos ainda por sedimentos e todo tipo de vestígios culturais como esqueletos humanos, ossos de animais, principalmente de peixes, restos de crustáceos, sementes e coquinhos, além de fogueiras, artefatos ósseos (pontas, anzóis, agulhas, adornos), líticos (lâminas de machados, quebra-coquinhos, almofarizes, lascas de quartzo, material lítico bruto utilizado) e malacológicos (adornos, anzóis, raspadores) e, em alguns casos, nas camadas superficiais, fragmentos de ceramica.

Segundo Gaspar et al. (2008), os grupos sambaquieiros apresentavam como característica fundamental a acumulação de restos de alimentos, em sua grande maioria as carapaças de moluscos, que foram estruturando paulatinamente plataformas que atualmente se destacam da paisagem atual. Os sambaquis foram construídos em locais estratégicos do ponto de vista dos recursos, notadamente enseadas, lagunas, estuários e manguezais. Considerando estes locais como pontos de concentração de sambaquis, Lima (1999-2000) e Gaspar (2004) observam que essas populações dispunham de grande diversidade e abundancia de recursos aquáticos, favorecendo a criação de assentamentos mais estáveis que, segundo Gaspar et al. (2008), possibilitou o surgimento de maior complexidade social.

Schell-Ybert $(2000,2001)$ tem demonstrado que a coleta de vegetais representou uma importante atividade entre os grupos sambaquieiros, sendo a Mata de Restinga e a Mata Atlântica, ambientes explorados frequentemente. Prous (1992) ainda faz referência à exploração dos afloramentos do Embasamento Cristalino, do qual exploravam matérias primas para a confecção de artefatos líticos. Devemos observar, ainda, que os seixos disponíveis na planície costeira constituíam a mais próxima e abundante fonte de recursos líticos para os grupos sambaquieiros, tendo alta frequência nos sambaquis. Contudo, Barbosa-Guimarães (2011) observa que a proximidade de fontes minerais não era fator que influenciava o assentamento dos grupos sambaquieiros.

Portanto, a escolha do local de assentamento era influenciada por fatores ambientais, principalmente pela proximidade de pontos de intersecções ambientais onde a previsibilidade, diversidade e abundancia dos recursos aquáticos minimizava a mobilidade dos grupos sambaquieiros. Devemos observar, contudo, que outros fatores de ordem cultural certamente eram considerados quando da escolha do local de assentamento, possivelmente estando relacionados aos locais de memória ancestral (Barret et al. 1991).

\section{Metodologia e Resultados}

Com base nos dados sobre a instalação dos sambaquis brasileiros e de porte dos estudos evolutivos para o Holoceno no Litoral Norte do Estado da Bahia (Martin e Dominguez 1992, Dominguez et al. 1999), foram realizadas prospecções arqueológicas nesta região (Silva 2000), bem como no litoral do Estado de Sergipe (Amancio 2001).

A base metodológica foi a construção de modelo arqueológico preditivo com base em dados geomorfológicos e geológicos (Allen et al. 1990; Lock e Stancic 1995). Este modelo associou dados da paleopaisagem do litoral Norte do Estado da Bahia e do litoral do Estado de Sergipe aos dados arqueológicos disponíveis sobre a implantação de sambaquis na paisagem. Assim, buscou-se verificar a existência de condições ambientais favoráveis à instalação de populações pescadoras-coletoras pré-coloniais durante o Holoceno Médio em parte do litoral da Região Nordeste do Brasil.

Um modelo de previsão é uma ferramenta que pode ser usada na determinação da probabilidade relativa de se identificar sítios arqueológicos em determinadas áreas pré-estabelecidadas. 
A modelagem preditiva é definida como um conjunto simplificado de hipóteses testáveis, com base em suposições, comportamentos ou em correlação empírica, que em uma tentativa de mínimos busca prever os loci das atividades humanas pretéritas, resultando na deposição de artefatos ou alteração da paisagem (Kohler e Parker 1986).

Um modelo testado é uma hipótese, uma previsão do que o pesquisador, com base em estudo e análise, acha que deve ser o padrão de eventos no futuro. Com isto em mente, o teste real da hipótese é celebrado sob a forma de levantamento de campo. Quando os sítios são localizados podem ser armazenados em uma base de dados que pode expandir e melhorar o modelo original. Esta informação pode ser posteriormente transferida para mapas de sensibilidade em uma tentativa de prever as áreas de probabilidade de alta, média e baixa para os sítios arqueológico.

O modelo de previsão de sítios utilizado nesta pesquisa foi um modelo indutivo, formado por meio dos dados coletados em campo associado aos dados paleoambientais.

\section{Prospecção no litoral norte do Estado da Bahia}

A área de estudo refere-se ao Município do Conde, localizado na latitude $11^{\circ} 48^{\prime} 49^{\prime \prime}$ Sul e a uma longitude $37^{\circ} 36^{\prime} 38^{\prime \prime}$ Oeste, estando a uma altitude de 12 metros (Figura 1).

De acordo com estudos efetuados por Domingues et al. (1999) no litoral do município do Conde, pode-se observar que, em resumo, a evolução da área se deu em três estágios (Figura 2).

Inicialmente, a cerca de 5.600 anos cal AP, durante a Última Transgressão, o Nível Relativo do Mar (NRM) encontrando-se a cerca de $5 \mathrm{~m}$ acima do atual, afogou as terras úmidas da atual planície costeira do Conde. Tal processo resultou na formação de um sistema de Ilha Barreira/Laguna. Após a Última Transgressão, iniciou-se uma regressão marinha com formação de um delta intralagunar formado pela desembocadura do rio Itapicuru, dando continuidade ao abaixamento do nível do mar, que se seguiu ao máximo da Última Transgressão. A paisagem lagunar foi paulatinamente substituída por pântanos de água doce e brejos, até chegar às condições atuais. Atualmente, a feição da costa encontra-se representada por uma vasta extensão de terrenos baixos e úmidos, relacionados aos rios Itapicuru e Curumaí, bordejada por depósitos Quaternários e Terciários.

A prospecção arqueológica identificou um sambaqui, denominado Ilha das Ostras, que foi formado durante a Última Transgressão, quando ocorreu a formação de uma área lagunar na região, que perdurou possivelmente até a data de 3.500 anos cal AP (Figura 3).

Nas sondagens exploratórias realizadas no sambaqui Ilha das Ostras foram identificados vestígios zooarqueológicos associados ao ambiente estuarino, bem como adornos confeccionados a partir de ossos e conchas, ossos humanos, artefatos líticos (brutos utilizados) e fragmentos cerâmicos (Quadro 1) (Silva 2000, Amancio-Martinelli 2006, 2007).

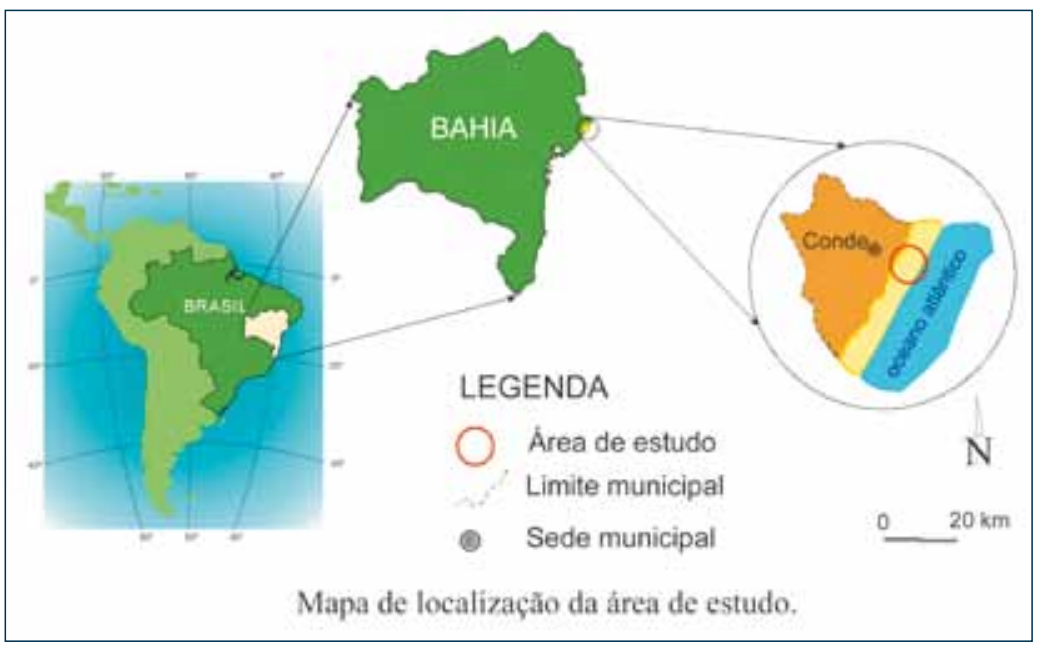

Figura 1. Localização da área de pesquisa, litoral Norte do estado da Bahia. 


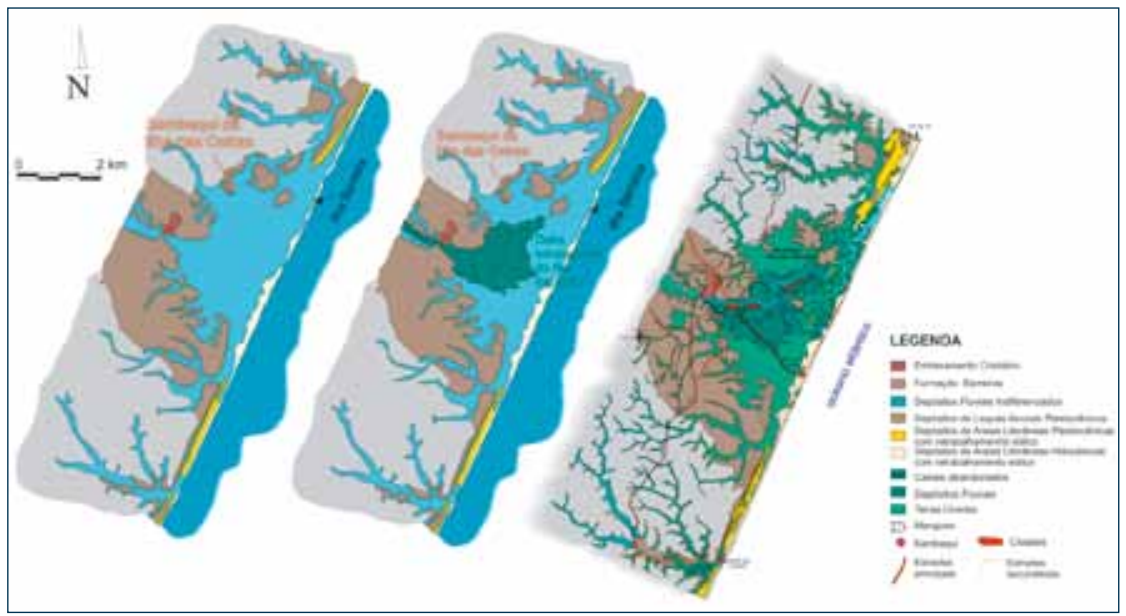

Figura 2. Evolução costeira no município de Conde - Bahia (Dominguez et al 1999).

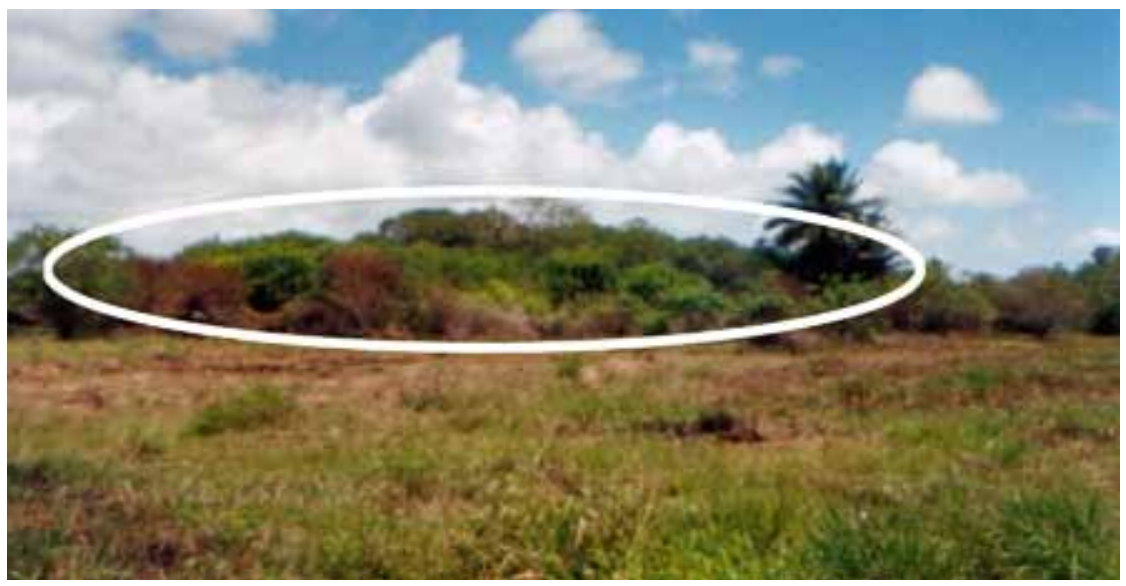

Figura 3. O sambaqui Ilha das Ostras, Conde, Bahia (Foto: S. Amancio-Martinelli).

Quadro 1. Identificação taxonômica dos vestígios zooarqueológico de habitat estuarino

\section{IDENTIFICAÇÃO TAXONOMICA}

MOLUSCA
BIVALVIA
Chrassostrea rhizophorae (Guilding,1828)
Lucina pectinata (Gmelin,1791)
Pugilina morio (Linneaus,1758)
Mytilla falcata (D’Orbigny, 1842)
CRUSTACEA
Ucides cordatus (Linnaeus, 1763)
Callinestes sp.
PISCES
OSTEICHTHYES
Tachysurus sp
Scianidae sp
CHONDRICTHYES
Squalomorphii
Batoidea

\section{Prospecção no litoral do Estado de Sergipe}

Dando prosseguimento à aplicação do modelo arqueológico preditivo, tendo como base a história evolutiva da região, durante o Holoceno, foi realizada prospecção arqueológica no litoral do Estado de Sergipe (Amancio 2001) (Figura 4).

Assim, o modelo indutivo arqueológico preditivo, de cunho geológico e geomorfológico, foi também aplicado ao litoral de Sergipe, considerando o pressuposto de que nas zonas costeiras, onde as condições lagunares não subsistiram por muito tempo durante o Holoceno, os sambaquis tenderiam a apresentar pequenas dimensões, não se destacando na paisagem (Silva 2000).

Segundo Bittencourt et al. (1983), durante a Última Transgressão por volta de 5.600 anos cal 


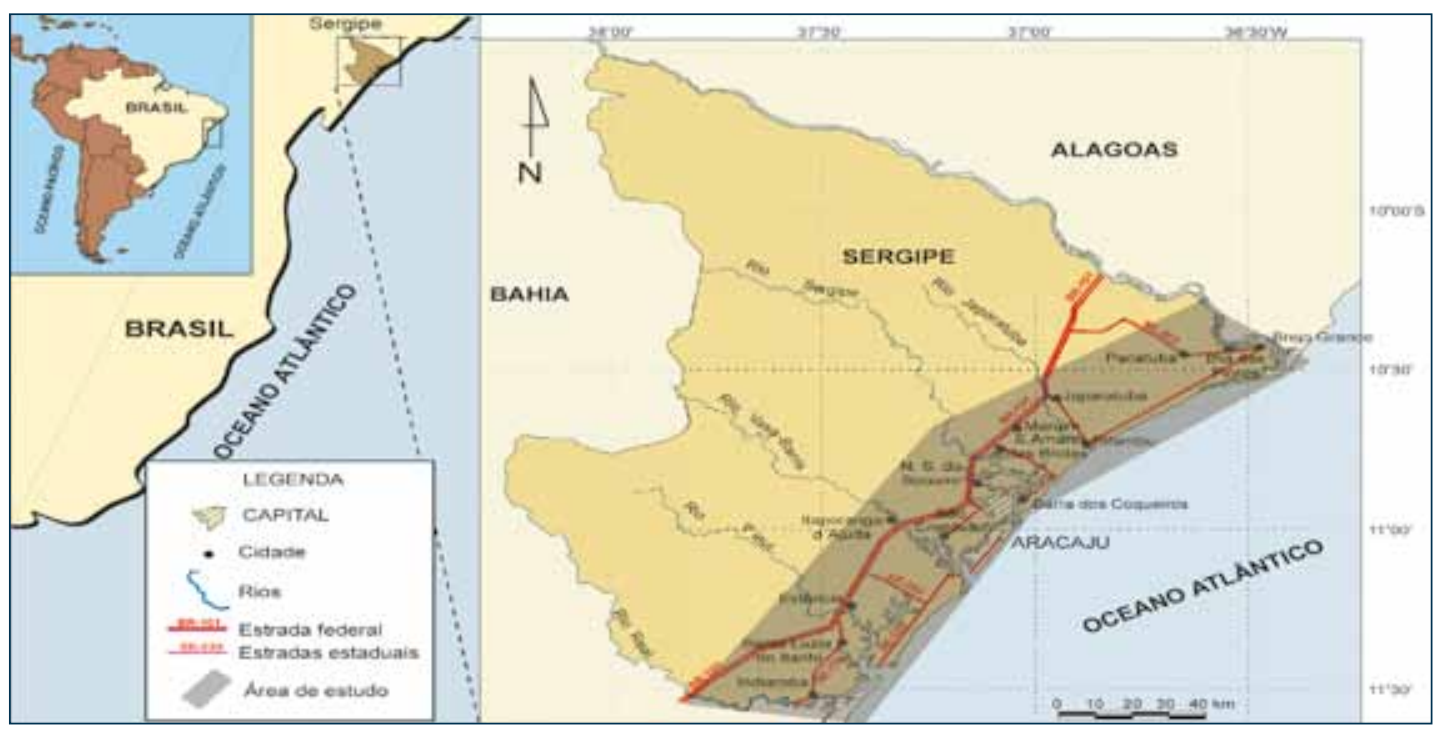

Figura 4. Localização da área de pesquisa - costa litorânea do Estado de Sergipe. (Amancio 2001).

AP, ocorreu a erosão parcial dos Terraços Marinhos Pleistocênicos. Ainda durante esse momento, em alguns trechos desse litoral o mar retrabalhou as falésias pertencentes ao Grupo Barreiras. Nesta época, o baixo curso dos rios que deságuam atualmente na região costeira passou a desaguar no interior de corpos lagunares originados pelo afogamento das terras baixas (Figura 5). Sobre esse episódio Bittencourt et al. (1983) reportam que "essas lagunas foram construídas ou a partir do afogamento da parte inferior dos vales entalhados na
Formação Barreiras e da rede de drenagem instalada nos Terraços Marinhos Pleistocênicos durante o evento anterior, ou ainda, mediante a formação de ilhas-barreiras".

Durante a regressão que se seguiu à Última Transgressão, o modelado da zona costeira adquiriu seus contornos atuais. Foram formados os Terraços Marinhos Holocênicos, dispostos externamente aos Terraços Marinhos Pleistocênicos e as lagunas perderam a sua comunicação com o mar, evoluindo para pântanos (Bittencourt et al. 1983) (Figura 6).

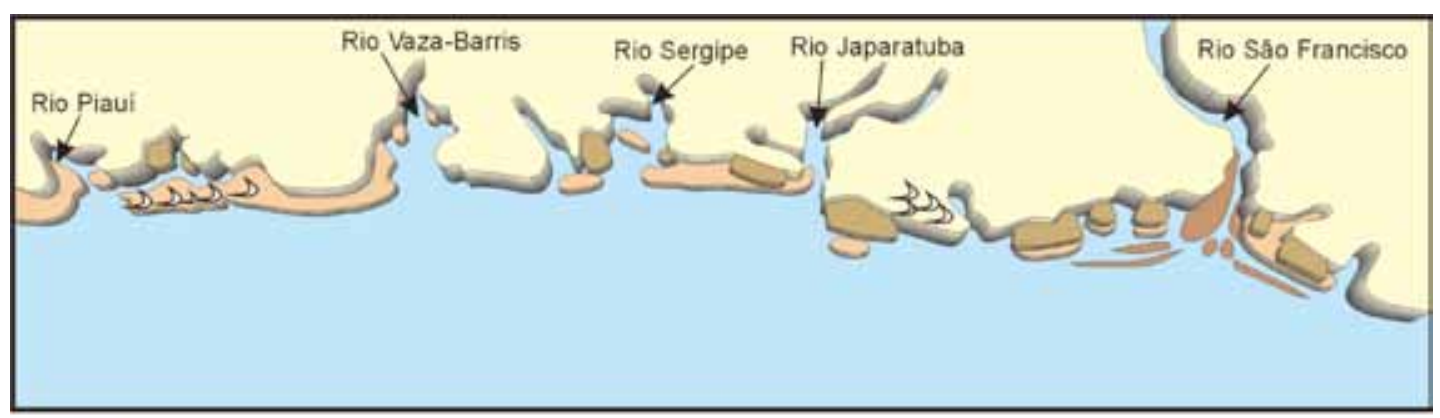

Formaçăo Barreiras

Leques aluviais coalescentes pleistocênicos

\section{D] Campos de dunas \\ Terraços marinhos pleistocênicos \\ Depósitos flúvio-lagunares}

Figura 5. Paleogeografia da zona costeira sergipana por volta do máximo da Última Transgressão 5.100 anos AP (Modificado de Bittencourt et al. 1983). 


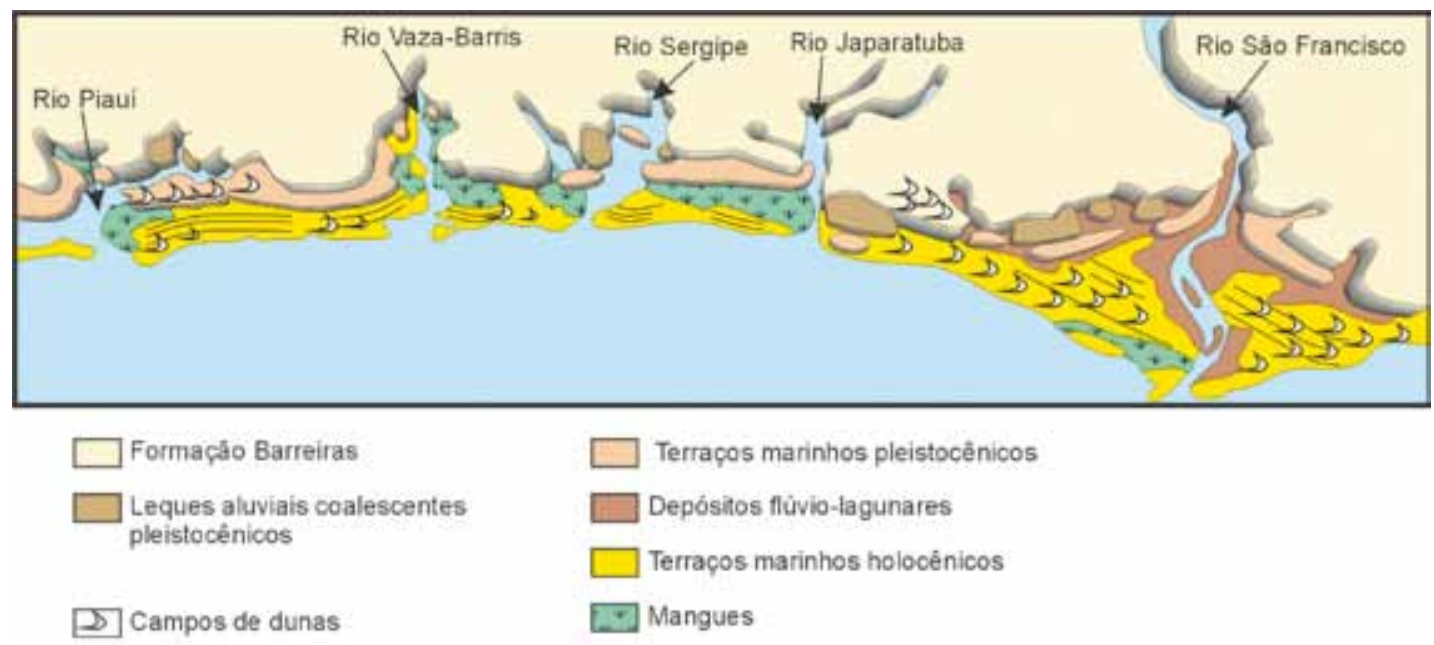

Figura 6. Morfologia esquemática atual da zona costeira sergipana (modificado de Bittencourt et al. 1983).

Baseado no mapa geológico do Estado de Sergipe (CPRM 1997) e no modelo evolutivo para o Quaternário proposto por Bittencourt et al. (1983) para a zona costeira do Estado de Sergipe, foi realizado um levantamento das áreas protegidas da zona costeira que possibilitassem um aproveitamento do ambiente para a caça, a coleta e a pesca, por grupos pré-coloniais durante os últimos $5.600 \mathrm{cal}$ anos AP (Amancio 2001).

A seleção dessas áreas a serem prospectadas se deu a partir de estudos geológico-geomorfológicos da região, uma vez que as zonas baixas, atualmente denominadas de terras úmidas, possivelmente teriam constituído ambientes protegidos do tipo baías/lagunas/ estuários, durante o máximo da Última Transgressão.

No litoral de Sergipe, a partir de critérios geológico-geomorfológicos e arqueológicos, os locais considerados como propícios à ocupação de grupos sambaquieiros são os depósitos pleistocênicos e holocênicos associados às planícies costeiras relacionadas às desembocaduras dos rios Japaratuba, Sergipe, Vaza-Barris e Piauí/Real.

A prospecção arqueológica realizada nestes alvos selecionados como mais favoráveis, a partir dos critérios geológico-geomorfológicos, não resultou na identificação de sítios arqueológicos do tipo sambaqui.

\section{Discussões e Conclusão}

Estudos arqueológicos assistemáticos desenvolvidos ao longo do século XX demonstra a presença recorrente de uma sequencia cerâmica na composição dos sambaquis setentrionais associado ao conjunto de vestígios arqueológicos recorrentes às ocupações dos grupos sambaquieiros identificados no litoral sul-sudeste do Brasil (Calderón 1969, 1973, 1974; Lopes 1924; Machado et al. 1991, Neves e Neves 2011). O início dos estudos sistemáticos, embora pontuais, se deu apenas, e de forma descontínua, a partir da segunda metade do século XX, tendo tido contribuição de Calderón (1964), para o litoral da Bahia, e Bandeira (2006, 2008), para o litoral do Maranhão. Estudos geomorfológicos, tendo os sambaquis com objeto de estudos, também foram desenvolvidos por Suguio et al. (2003), para o litoral do Rio Grande do Norte.

Esta rara e pontual produção bibiográfica demonstra o caráter praticamente inédito de pesquisas sobre os sambaquis do Nordeste. Dois fatores podem estar contribuindo para tal quadro de pesquisas: um primeiro relacionado a não colonização de várias porções do litoral da Região Nordeste, notadamente da faixa entre os estados de Sergipe e Ceará, exceção da costa de Alagoas, por populações sambaquieiras influenciada pela formação geológica e geomorfológica que não propiciou a formação de ambientes favoráveis à fixação destas populações; e um segundo fator, relacionado ao baixo investimento nas pesquisas arqueológicas sistemáticas que propiciem a formação de quadros regionais detalhados do ponto de vista sociocultural e paleoambiental (Amancio 2001). 
No caso do Estado da Bahia, no litoral de Conde foi identificado o sambaqui Ilha das Ostras. A localização e disposição do sítio na área do paleoestuário indicam que existiram locais privilegiados para implantação do assentamento e que, possivelmente, a escolha desses locais se deu em decorrência da alta produtividade, considerando que o sambaqui se localiza em frente a maior extensão estuarina. A presença dos rios Itapicuru e Curumaí é outro fator a atuar localmente, conferindo, juntamente com os movimentos de maré, um maior fluxo de nutrientes e renovação da água na área de maior extensão estuarina.

O estudo realizado na paleobaía do Conde também revela que o grupo que ocupou o sambaqui Ilha das Ostras implantou-se sobre ilha ou península inseridas no interior da maior extensão paleoestuarina. Isto indica não só a preferencia pela implantação na porção mais ampla da baía, como também o entorno estar ocupado pelo manguezal.

Em relação ao litoral do Estado de Sergipe este não condicionou geologicamente a formação de grandes ambientes lagunares nos últimos 5.000 anos AP. Uma das razões para a não formação desses ambientes seria possivelmente uma declividade acentuada da planície costeira sergipana. Roy et al (1994) sugerem que planícies costeiras com declividades superiores a $0,7^{\circ}$ não favorecem a formação de sistemas ilhas barreiras/lagunas, suporte este que necessita ser ainda testado para a costa de Sergipe (Amâncio e Dominguez 1999).

Pôde-se constatar que as zonas baixas que separam os Terraços Marinhos Holocênicos dos Terraços Marinhos Pleistocênicos no litoral sergipano, além de muito estreitas, nunca constituíram lagunas no máximo da Transgressão Holocênica. Estas são apenas zonas topograficamente mais baixas onde o lençol freático aflora formando terras úmidas de água doce, ou então foram originalmente muito estreitas e depois ocupadas por canais de rios que, como resultado de meandramento lateral as fizeram aumentarem de largura (Amâncio e Dominguez 1999). Um exemplo claro é a planície costeira de Abaís (entre o rio Piauí e o rio Vaza-Barriz), onde o substrato da zona baixa que separa os terraços marinhos holocênicos dos pleistocênicos é arenoso, e não apresenta qualquer indício da existência de uma antiga laguna.

Outro aspecto a ser levado em conta é que os grandes estuários associados aos rios que deságuam na costa sergipana tinham no máximo da Última Transgressão provavelmente, suas áreas intermareais bastante reduzidas. As paredes íngremes dos vales destes rios escavados no Grupo Barreiras não favoreceram a formação de áreas intermareais significativas durante períodos do nível relativo do mar mais alto que o atual. Portanto, no máximo da Última Transgressão não haveria condições para o manguezal se implantar, uma vez que a água estava encostada diretamente nas paredes, chegando até a erodi-las. Isto dificultaria a formação de planícies de marés e de manguezais, ou estes teriam dimensões bastante reduzidas.

Portanto, se existiram grupos pré-históricos nesse período na planície costeira de Sergipe, esses não utilizaram de modo extensivo recursos extraídos de ambientes lagunares/estuarinos, uma vez que a zona costeira não apresentou durante o máximo da Última Transgressão uma fisiografia que favorecesse o aparecimento destes ambientes.

$\mathrm{O}$ modelo preditivo arqueológico adotado na pesquisa demonstrou sua validade ao identificar no litoral norte do Estado da Bahia um sambaqui implantado no paleoestuario, criando condições favoráveis para a colonização da região por grupos sambaquieiros. Da mesma forma, mas em sentido contrário, a sua aplicação no litoral do Estado de Sergipe indicou uma ausencia de sítios sambaquis durante o Holoceno Médio e Tardio, lacuna associada à inexistência de ambientes propícios à ocupação sambaqueira.

\section{Referências Citadas}

\footnotetext{
Allen, K.M.S.; S.W. green and E.B.W. Zubrow 1990 Interpreting Space: GIS and Archaeology, Taylor and Francis, London, UK.

Amancio, S.G.

2001 Influência da Evolução Costeira Holocênica na Ocupação por Grupos PCC no Litoral de Sergipe. Dissertação de Mestrado. Programa de Pós-Graduação em Geologia, Universidade Federal da Bahia, Salvador.
}

Amancio-Martinelli, S.

2006 Os artefatos do sambaqui Ilha das Ostras no litoral norte do estado da Bahia, Workshop Arqueológico de Xingó, IV, Aracaju. Anais, p. 122-124. MAX/PETROBRAS.

2007 Processo de Formação do Sambaqui Ilha das Ostras no Litoral Norte do Estado da Bahia. Programa de PósGraduação em Geologia, Universidade Federal da Bahia, Salvador. 
Amancio, S.G; J. e M.L. Dominguez

1999 Influencia da Evolução Costeira Holocênica e a Ocupação Pré-histórica no Litoral de Sergipe. Congresso da Associação Brasileira de Estudos do Quaternário-ABEQUA, VII, Porto Seguro. Anais. CD-Room.

Barbosa-Guimarães, M.

2011 O modelo arqueológico de mudança sociocultural para a faixa litoranea do Estado do Rio de Janeiro, Arqueologia do Rio de Janeiro (J. Cordeiro - editor), Rio de Janeiro, Laboratório de Arqueologia Brasileira, p. 45-68.

2012 Landscape archaeology in coastal areas: technology and subsistence among prehistoric populations, Scientia Plena, $N^{\circ}$ 8: 1-9, Aracaju, Sociedade Sergipana de Ciencias.

Bandeira, A.M.

2006 O sambaqui do Bacanga na Ilha de São Luis- Maranhãoinserção na paisagem e levantamento extensivo, Canindé, MAX/UFS, Canindé de São Francisco, vol. 8, № 8: 43-66.

Bandeira, A.M. e M.L.A.F. Pacheco

2007 A Arqueofauna de vertebrados do sambaqui do Bacanga-São Luís-Maranhão: áreas de captação de recursos e identificação preliminar dos taxa, Canindé, MAX/UFS, Canindé de São Francisco, vol. 10, N 1: 143-168.

Barrett, J.; R. Bradley and M. Green

1991 Landscape, Monument and Society: The Prehistory of Cranhorne Chase, Cambridge University Press, Cambridge, UK.

Bittencourt, A.C. S.P.; L. Martin; G.S. Vilas Boas and J-M Flexor 1979 Quaternary marine formations of the coast of the State of Bahia, Brazil. International Symposium of Coastal Evolution in the Quaternary (Suguio K., T. R. Fairchild., L. Martin and J-M. Flexor, editores), Proccedings, São Paulo, p. 232-253.

Bittencourt, A.C.S.P.; L. Martin; J.M.L. Dominguez e Y.M.A. Ferreira

1983 Evolução Paleográfica Quaternária da Costa do Estado de Sergipe e da Costa Sul do Estado de Alagoas, Revista Brasileira de Geociências, São Paulo, vol. 13, Nº 2: 93-97. Calderón, V.

1964 O Sambaqui da Pedra Oca, Relatório de uma Pesquisa, 2, Universidade da Bahia, Instituto de Ciências Sociais, $88 \mathrm{p}$.

1969 Nota prévia sobre a arqueologia das regiões Centra, e Sudoeste do Estado da Bahia. Publicações Avulsas, Belém, Museu Paraense Emílio Goeldi, No 10: 135-146.

1973 A pesquisa arqueológica nos estados da Bahia e Rio Grande do Norte, Dédalo, Instituto de Pré-História, São Paulo, vol. 9, No 18: 25-31.

1974 Contribuição para o conhecimento da arqueologia do recôncavo e do Sul da Bahia, Publicações Avulsas, Belém, Museu Paraense Emílio Goeldi, Nº 26: 141-155.

CPRM

2007 Mapa Geológico do Estado de Sergipe.

Deblasis, P.; A. Kneip, R. Scheel-Ybert, P.C.F. Giannini e M.D. Gaspar

2007 Sambaquis e paisagem: dinâmica natural e arqueologia regional no litoral do Sul do Brasil, Arqueología Suramericana, São Paulo, Museu de Arqueologia e Etnologia, No 3: 29-61.

Dominguez J.L.

1987 Quaternary sea level changes and the depositional architeture of beach-ridge strandplains along the coast of Brazil. Ph.D. Dissertation, University of Miami-Florida/USA.
Dominguez, J.M.L.; AC.S.P. Bittencourt e L. Martin

1981 Esquema evolutivo da sedimentação quaternária nas feições deltaicas dos rios São Francisco (SE/AL), Jequitinhonha (BA), Doce (ES) e Paraíba do Sul (RJ). Revista Brasileira de Geociências, São Paulo, vol. 11, $\mathrm{N}^{\circ}$ 4: 227-237.

Dominguez, J.M.L.; Z.M.N. Leão e R.S. Lyrio

1996 Litoral Norte do Estado da Bahia: evolução costeira e problemas ambientais. Relatório Final. Roteiro de Excursão do Congresso Brasileiro de Geologia, SBG-Núcleo Bahia/ Sergipe, Salvador.

Dunbar, J.S.; S.D. Webb and M. Faugght

1991 Inundated Prehistoric Sites in Apalachee Bay, Florida, and the Search for the Clovis Shoreline, Paleoshorelines and Prehistory: an Investigation of Method (L.L. Johnson - editor), Boca Raton, Ann Harbor, London, CRC Press, Chapter 6: 117-149.

Erlandson, J.M.

2010 Early Hunter-Gatherers of the California Coast, New York, London, Plenum Press.

Figuti L.

1993 O Homem Pré-Historico, o Molusco e o Sambaqui: Considerações Sobre a Subsistência dos Povos Sambaquieiros, Revista do Museu de Arqueologia e Etnologia, $N^{\circ} 3:$ 67-80, São Paulo, MAE/USP.

Gaspar, M.D.

2004 Aspectos da Organização de um Grupo de Pescadores, Coletores e Caçadores: Região Compreendida entre a Ilha Grande e o Delta do Paraíba do Sul, Estado do Rio de Janeiro, Pesquisas, Instituto Anchietano de Pesquisa, São Leopoldo, $\mathrm{N}^{\circ}$ 59: 1-163.

Gaspar, M.D.; P. Deblasis; S.K. Fish and P.R. Fish

2008 Sambaqui (Shell Mound) Societies of Coastal Brazil, Handbook of South American Archaeology (E. Silverman \& W.H. Isbell- editors), Springer, New York, p. 319-338.

Jardine, W.G. and A. Morrison

1976 The archaeological significance of Holocene coastal deposits in south-western Scotland, Geoarchaeology Earth Science and the Past, Section 2 Coastal and Lacustrine environments, Gerald Duckworth and Co. Ltd., London, p. 175-196.

Kohler, T.A. and S.C. Parker

1986 Predictive Models for Archaeological Resource Location, Advances in Archaeological Method and Theory, Arizona, Arizona University Press, No 9: 397-452.

Lima, T.A.

1999-2000 Em busca dos frutos do mar: os pescadorescoletores do Centro-Sul do Brasil, Revista da USP, São Paulo, USP, $\mathrm{N}^{\circ}$ 44: 270-327.

Lock, G. and Z., Stancic

1995 Archaeology and Geographical Information Systems: A European Perspective, London, Taylor \& Francis.

Lopes, R.

1924 A civilização lacustre do Brasil, Boletim do Museu Nacional, Rio de Janeiro, Museu Nacional, vol. 1, No 2 .

Machado, A.L.; G.C. Conceição e D.F. Lopes

1991 Os sambaquis da Ilha de São Luís, Maranhão. I Simpósio de Pré-História do Nordeste Brasileiro, Recife, Pernambuco. Anais.

Martin, L. and J.M.L. Domínguez

1992 Geological history of coastal lagoons-with particular references to examples from the east coast of Brazil, 
Coastal Lagoon Processes (B. Kjerfve - editor), Amsterdam, Elsevier.

Neves, S.M. e M.M. Neves

2011 Ocupação humana e a probabilidade da presença se sítios arqueológicos do tipo sambaqui no litoral da Paraíba, Revista de Geografia, Recife, No 27: 277-288.

Prous, A.

1992 Arqueologia Brasileira. Brasília, Editora UNB.

Roy, P.S.; P.J. Cowell; M.A. Ferland and B.G. Thom 1994 Wave dominated coasts, Coastal Evolution (Carter, R.W.G. and C.D. Woodroffe, - editors), Cambridge, Cambridge University Press, p. 121-186.

Sandweiss, D.

2008 Early Fishing Societies in Western South America, Handbook of South American Archaeology (E. Silverman and W.H. Isbell - editors), Springer, New York, p. 145-156. Scheel-Ybert, R.

2000 Vegetation stability in the Southern Brazilian coastal are from 5500 to $1400 \mathrm{yr}$ BP D-deduced from charcoal analysis, Review of Palaeobotanic and Palynology $N^{\circ} 110$ : 11-138. 2001 Man e vegetation in Southeastern Brazil during the Late Holocene, Journal of Archaeological Science No 28: 471-480.

Scheel-Ybert, R.; M.C. Afonso; M. Barbosa-Guimarães; M.D. Gaspar e J-P. Ybert

2009 Considerações sobre o papel dos sambaquis como indicadores do nível do mar, Quaternary Environmental Geoscience, vol. 1, No 1: 3-9.

Silva C.C.

2000 Herança Geológica como ferramenta para a prospecção de sambaquis no litoral norte do estado da Bahia: o exemplo do sambaqui Ilha das Ostras. Dissertação de Mestrado. IGEO/UFBA. Salvador-BA.

Suguio, K.; L. Martin; A.C.S.P. Bittencourt; J.M.L. Domínguez,

J-M. Flexor e A.E.G. Azevedo

1985 Flutuações do nível do mar durante o Quaternário superior ao longo do litoral brasileiro e suas implicações na sedimentação costeira, Revista Brasileira de Geociências, São Paulo, vol. 15, No 4: 273-286.

Suguio, K.; L. Martin and J-M, Flexor

1991 Paleoshorelines and the sambaquis of Brazil, Paleoshorelines and Prehistory: an Investigation of Method (L. L. Johnson - editor), CRC Press, Boca Raton Ann Arbor, London, Chapter 4.

Suguio, K.; A.M. F. Barreto; F.H.R. Bezerra e L.C.R. Pessenda 2003 Idades ao radiocarbono de prováveis sambaquis do litoral Nordeste Brasileiro, IX Congresso da Associação Brasileira de Estudos do Quaternário, Recife, Pernambuco, Anais.

Tenório, M.C.; K. Swett; F. Ferreira-Dias; J. Wagner de Alencar;

R.C.C.L. Souza, M. Barbosa-Guimarães e C. Ribeiro

2010 The site Geribá II, Buzios, Rio de Janeiro. Understanding an Ancient Shell Mound from Archaeological, Zooarchaeological and Geological Perspectives, 11th International Council for Archaeozoology Conference, 2010, Paris, Proceedings, Paris, (Christine Lefèvre - editor), BAR International Series $\mathrm{N}^{\circ} 1: 257-265$

Villagrán, X.S.; D. Klokler, S. Peixoto, P. Deblasis and P.C.F. Giannini

2011 Building Coastal Landscapes: Zooarchaeology and Geoarchaeology of Brazilian Shell Mounds, The Journal of Island and Coastal Archaeology, $\mathrm{N}^{\circ}$ 6: 211-234. 
